\title{
Schriften von Martine Dalmas
}

\section{Wissenschaftliche Publikationen}

\subsection{Qualifikationsschriften}

1982 Dissertation: Étude de quelques stratégies discursives en allemand. Lyon, Université Lyon-2.

1993 Habilitationsschrift: Procédés argumentatifs en allemand. Évaluation et argumentation dans l'élaboration et la structuration du discours en allemand. Paris, Université Paris IV-Sorbonne.

\subsection{Mitherausgeberschaften}

1987 Das Passiv im Deutschen. Tübingen: Niemeyer (in Zusammenarbeit mit dem C.R.L.G.).

1994 Partizip und Partizipialgruppen im Deutschen. Tübingen: Narr (in Zusammenarbeit mit Daniel Bresson).

1998 Grenzsteine und Wegweiser. Textgestaltung, Redesteuerung und formale Zwänge. Festschrift für Marcel Pérennec zum 60. Geburtstag. Tübingen: Stauffenburg (in Zusammenarbeit mit Roger Sauter).

2004 Énoncer. L'ordre informatif dans les langues. Paris: L'Harmattan (in Zusammenarbeit mit Pierre Cotte und Hélène Wlodarczyk).

2006 Text und Sinn. Studien zur Textsyntax und Deixis im Deutschen und im Französischen. Festschrift für Marcel Vuillaume zum 60. Geburtstag. Tübingen: Stauffenburg (in Zusammenarbeit mit Jean-François Marillier und Irmtraud Behr).

2009 Wissenschaftliche Textsorten im Germanistikstudium deutsch-italienisch-französisch kontrastiv. Pisa: Servizio Editoriale Universitario di Pisa (in Zusammenarbeit mit Marina Foschi Albert und Eva Neuland).

2010 Germanistische Linguistik extra muros - Aufforderungen. (= Linguistische Treffen in Wrocław, Bd. 5). Wrocław: Atut - Dresden: Neisse Verlag 
(in Zusammenarbeit mit Iwona Bartoszewicz, Joanna Szczęk und Artur Tworek).

2011 Déconstruction - Reconstruction. Autour de la pensée de Jean-Marie Zemb. Limoges: Lambert-Lucas (in Zusammenarbeit mit Thierry Gallèpe).

Text und Stil im Kulturvergleich. München: Iudicium (in Zusammenarbeit mit Marina Foschi Albert, Marianne Hepp und Eva Neuland).

2014 Figurative Sprache - Figurative Language - Langage figuré. Festgabe für Dmitrij O. Dobrovol'skij. Tübingen: Stauffenburg (in Zusammenarbeit mit Elisabeth Piirainen).

2015 Variation im europäischen Kontrast - Untersuchungen zum Satzanfang im Deutschen, Französischen, Italienischen, Norwegischen, Polnischen und Ungarischen. Berlin/Boston: De Gruyter (in Zusammenarbeit mit Cathrine Fabricius-Hansen und Horst Schwinn).

\subsection{Artikel}

1981 Savoir parler et savoir discourir - Quelques aspects de la 'rhétorique socialiste'. In: L’argumentation. Lyon: Presses universitaires de Lyon. 179-201.

Sogar die Linguisten wissen nicht, was sie sagen. In: Weydt, Harald (Hg.) (1981): Partikeln und Deutschunterricht. Heidelberg: Julius Groos. 165-176 (in Zusammenarbeit mit Henri Rünneburger).

1982 Orienter ou diriger un dialogue? Fonction argumentative de quelques opérateurs en allemand et en français. In: Contrastes 4-5. 165-176.

1983 nämlich et und zwar. In: Cahiers d’Études Germaniques 7. 121-151 (in Zusammenarbeit mit Marius E. Granito).

1984 nämlich et und zwar. In: Cahiers d'Études Germaniques 8. 165-209 (in Zusammenarbeit mit Marius E. Granito).

1985 Les particules dans le dialogue: la notion de pertinence. In: Questions linguistiques à l'agrégation d’allemand. Metz: Université de Metz. 121-141.

1986 Marques linguistiques de l'interaction verbale. In: Cahiers d’Études Germaniques 12. 145-159. 
Le choix entre sein et werden dans les phrases passives (I). In: Nouveaux Cahiers d'Allemand 2. 147-170 (in Zusammenarbeit mit Jean-Marc Bobillon, Jean-François Marillier und Marcel Vuillaume).

Le choix entre sein et werden dans les phrases passives (II). In: Nouveaux Cahiers d'Allemand 3. 227-246 (in Zusammenarbeit mit Jean-Marc Bobillon, Jean-François Marillier und Marcel Vuillaume).

1987 Von außen betrachtet. Was ist am Deutschen so würzig?. In: Sprachreport 1. 11.

La demande d'information et de justification. Son marquage dans le dialogue. In: Cahiers d’Études Germaniques 15. 57-68.

1988 Bewertung im argumentativen Handeln. In: Cahiers d'Études Germaniques 14. 57-68.

Bewertung im argumentativen Handeln (2. Teil). In: Cahiers d'Études Germaniques 15. 17-27.

1989 Sprechakte vergleichen: ein Beitrag zur deutsch-französischen Partikelforschung. In: Weydt, Harald (Hg.) (1989): Sprechen mit Partikeln. Berlin: De Gruyter. 228-239.

Nebenbei gesagt, anscheinend überflüssig, aber eigentlich wichtig: übrigens und Co. In: Reiter, Norbert (Hg.) (1989): Akten des 23. linguistischen Kolloquiums Berlin 1988. Tübingen: Niemeyer. 423-430.

1990 Partikelforschung, konkret. In: Deutsch als Fremdsprache 5. 285-289.

1991 Zur Erweiterung des Begriffs 'Umstandsangabe'. In: Klein, Eberhard et al. (Hgg.) (1991): Akten des 24. linguistischen Kolloquiums, Universität Bremen 4.-6. September 1989. Tübingen: Niemeyer. 261-271.

Aspekte des Dialogs in 'Romulus der Große' von Friedrich Dürrenmatt. In: Chaiers d'Études Germaniques 20. 25-35.

Des pré-verbes non conventionnels?. In: Confais, Jean-Paul (Hg.) (1991): La linguistique à l'agrégation d'allemand 1991. Actes du colloque des linguistes germanistes. Toulouse: Université de Toulouse Le Mirail. 27-42.

1992 Bewertung durch Partikeln. In: Deutsch als Fremdsprache 2. 90-95.

1993 Nachgestelltes in der deutschen Verbalgruppe: Formen und pragmatische Funktionen. In: Marillier, Jean-François (Hg.) (1993): Satzanfang Satzende. Syntaktische, semantische und pragmatische Untersuchung zur Satzabgrenzung und Extraposition im Deutschen. Tübingen: Narr (= Eurogermanistik 3). 205-218. 
1994 Gedankenstriche: Zum Streichen oder Unterstreichen?. In: Cahiers d'Études Germaniques 27. 55-66.

1995 Was üblicherweise einer Frage vorausgeht. In: Schecker, Michael (Hg.) (1995): Fragen und Fragesätze im Deutschen. Tübingen: Narr. 257-269.

Semantische Reduktion und thematische Regression. Versuch einer funktionalen Beschreibung von jedenfalls. In: Faucher, Eugène/Métrich, René/Vuillaume, Marcel (Hgg.) (1995): Signans und Signatum. Auf dem Weg zu einer semantischen Grammatik. Festschrift für Paul Valentin. Tübingen: Narr. 265-277.

Tanz auf dem doppelten Boden der Tatsachen. Zu den diskursiven Funktionen von eigentlich. In: Popp, Heidrun (Hg.) (1995): Deutsch als Fremdsprache: An den Quellen eines Faches. Festschrift für Gerhard Helbig zum 65. Geburtstag. München: Iudicium. 217-227.

1996 La description des mots du discours. Pour une approche cognitive. In: Dupuy-Engelhardt, Hiltraud (Hg.) (1996): Questions de méthode et de délimitation en sémantique lexicale. Reims: Presses universitaires de Reims. 25-35.

Die sogenannten Nebensatz-Korrelate. Oder: Vertretungen nur als Mittel zur Rettung von Leerstellen?. In: Pérennec, Marie-Hélène (Hg.) (1996): Pro-formen des Deutschen. Tübingen: Stauffenburg. 23-34.

1997 Contextualisation des questions. In: Cahiers d’Études Germaniques 32. 201-214.

Sprechereinstellung und Hörerorientierung. Kognitiver Ansatz bei der kontrastiven Beschreibung der Gesprächswörter. In: Rovere, Giovanni/ Wotjak, Gerd (Hgg.) (1997): Studien zum romanisch-deutschen und innerromanischen Sprachvergleich. Frankfurt a.M. u.a.: Lang. 264-282.

1998 La fin et les moyens. In: Cahiers d'Études Germaniques 35. 145-162.

D’abord, et après? Le marqueur d'intégration français et ses cousins germains. In: Schnedecker, Catherine (Hg.) (1998): Les corrélats anaphoriques. Recherches linguistiques Vol. XXII, Université de Metz. Paris: Klincksieck. 75-95.

hinter et nach: problèmes d'orientation et question de perspective. In: Dupuy-Engelhardt, Hiltraud (Hg.) (1998): L'organisation lexicale et cognitive des dimensions spatiale et temporelle. Reims: Presses universitaires de Reims. 59-70.

Zuerst kommt das Fressen, dann... - oder: die Kunst, Prioritäten zu setzen. In: Dalmas, Martine/Sauter, Roger (Hgg.) (1998): Grenzsteine und Wegweiser. Textgestaltung, Redesteuerung und formale Zwänge. Fest- 
schrift für Marcel Pérennec zum 60. Geburtstag. Tübingen: Stauffenburg. 141-152.

1999 Nichtsdestoweniger bleibt das Verdienst... Zum Schlusswort in Rezensionen. In: Cahiers d'Études Germaniques 37. 76-88.

Wie gebettet, so geschlafen. In: Cortès, Colette/Rousseau, André (Hgg.) (1999): Catégories et connexions. Mélanges en l'honneur de Jean Fourquet pour son 100ème anniversaire. Lille: Presses universitaires de Lille. 199-214 (in Zusammenarbeit mit François Muller).

'Ende gut, alles gut.' Die französischen Konnektoren 'enfin' und 'finalement' im Sprachvergleich. In: Reinart, Silvia/Schreiber, Michael (Hgg.) (1999): Akten der gleichnamigen Sektion des ersten Kongresses des Franko-Romanistenverbandes (Mainz, 24.-26. September 1998). Bonn: Romanistischer Verlag. 189-208.

Fakten und Effekte: Wozu gebraucht man eigentlich 'tatsächlich' und Co? In: Skribiski, Bernd/Wotjak, Barbara (Hgg.) (1999): Linguistik und Deutsch als Fremdsprache. Festschrift für Gerhard Helbig zum 70. Geburtstag. Tübingen: Niemeyer. 53-66.

2000 Les particules et autres mots de la communication dans les formules et routines: degrés de tolérance. In: Gréciano, Gertrud (Hg.) (2000): Micro - et macrolexèmes et leur figement discursif. Études de linguistique comparée français - allemand. Peeters: Louvain/Paris. 347-361.

2001 Nackte Tatsachen auf frischer Tat ertappt. In: Heinrich, Wilma/Heiss, Christine (Hgg.) (2001): Modalità e Substandard. Bologna: CLUEB. 193-213.

Les circonstants en allemand. Incidences, dissidences et ... influences. In: Cercle Linguistique d'Aix (Hg.) (2001): Travaux 17, Adverbe et circonstant. Aix-en-Provence: Presses de l'Université de Provence. 101-119.

Empfehlen und Ablehnen in wissenschaftlichen Rezensionen. Versuch eines deutsch-französischen Vergleichs. In: Wotjak, Gerd (Hg.) (2001): Akten der IV. Arbeitstagung zum deutsch-romanischen Sprachvergleich. Frankfurt a.M. u.a.: Lang. 467-476.

Aspects de la lexicalisation du 'comitatif' en allemand. In: Dupuy-Engelhardt, Hiltraud (Hg.) (2001): La lexicalisation de structures conceptuelles. Reims: Presses universitaires de Reims. 145-159.

Routineformeln: ein willkommener Beitrag zur Heterogeneität der Rede. In: Burger, Harald/Häcki-Buhofer, Annelies/Gautier, Laurent (Hgg.) (2001): Phraseologia Amor. Aspekte europäischer Phraseologie. Hohengehren: Schneider Verlag. 63-72. 
Der Weisheit letzter Schluss... Zur Funktion des Schlusswortes in Rezensionen. In: Jakobs, Eva-Maria/Rothkegel, Annely (Hgg.) (2001): Perspektiven auf Stil. Tübingen: Niemeyer. 305-320.

Perspektivenwechsel durch Konnektoren und 'Formen mit konnektorähnlicher Funktion’. In: Cambourian, Alain (Hg.) (2001): Textkonnektoren. Tübingen: Stauffenburg. 109-127.

2002 Sens dessous-dessus. [Enseignement de la] grammaire et bon sens. In: Métrich, René/Petit, Jean (Hgg.) (2002): Didascalies - Analyses et réflexions en linguistique, textologie et didactique - Mélanges en l'honneur d'Yves Bertrand pour son soixante-dixième anniversaire. Nancy: Bibliothèque des Nouveaux Cahiers d’Allemand. 251-272.

2003 Le compte-rendu critique: entre débat et convenances. In: Robert, Valérie (Hg.) (2003): Intellectuels et polémiques dans l'espace germanophone. Asnières: PIA. 97-110.

Soweit das Auge reicht? Stand- und Blickpunkte durch sog. Modalisatoren. Ein Rückblick. In: Baudot, Daniel/Behr, Irmtraud (Hgg.) (2003): Funktion und Bedeutung. Tübingen: Stauffenburg. 197-208.

Französische Sichtweisen und folgenschwere Konsequenzen. In: Stickel, Gerhard (Hg.) (2003): Deutsch von außen. Jahrbuch 2002 des Instituts für Deutsche Sprache. Berlin/New York: De Gruyter. 111-127.

2004 L'ajout en allemand. In: Cotte, Pierre/Dalmas, Martine/Wlodarczyk, Hélène (Hgg.) (2004): Énoncer. L'ordre informatif dans les langues. Paris: L'Harmattan. 207-214.

Anpassung an die neuen Erfordernisse. In: Goltschnigg, Dittmar/Swob, Anton (Hgg.) (2004): Zukunftschancen der deutschen Sprache. Grazer Humboldt-Kolleg, Nov. 2002. Wien: Praesens. 140-143.

Wenn einer ruft... Ausrufe, Aufrufe und dergleichen Abgrenzung aufgrund der diskursiven Haltung. In: Krause, Maxi/Ruge, Nikolaus (Hgg.) (2004): Das war echt spitze! Zur Exklamation im heutigen Deutsch. Tübingen: Stauffenburg. 67-76.

2005 Dringend nötig: der „Blick über den Satzrand“. Zu den Schwierigkeiten von französischen Studierenden bei der Texterzeugung. In: Adamzik, Kirsten/Krause, Wolf-Dieter (Hgg.) (2005): Text-Arbeiten. Textsorten im fremd- und muttersprachlichen Unterricht an Schule und Hochschule. Tübingen: Narr. 97-110.

Der Widerspenstigen [erfolgreiche?] Zähmung. Partikeln und verwandte Widerspenstige im deutsch-französischen Vergleich. In: Schmitt, Christian/Wotjak, Barbara (Hgg.) (2005): Beiträge zum romanisch-deutschen 
und innerromanischen Sprachvergleich. Akten der gleichnamigen internationalen Arbeitstagung (Leipzig, 4.10.-6.10.2003), Bd. 1. Bonn: Romanistischer Verlag. 35-46.

2006 Jenseits der (Satz)grenze - syntaktische Zwänge und rhetorische Freiheiten. In: Kalba ir kontekstai - Mokslo darbai 1 (= Sprache in Kontexten - Linguistische Arbeitspapiere der Pädagogischen Universität Vilnius 1). 15-24.

'Entschuldigen Sie bitte, sind Sie Auslandsgermanistin?' Oder: Versuch einer Antwort auf eine falsch gestellte Frage. In: Deutsch als Fremdsprache 1/2006. 3-7.

Wie plattgesessene Currywürste und nassgepisste Klodeckel entstehen. Wahlverwandtschaften der 3. Art. In: Marillier, Jean-François/Dalmas, Martine/Behr, Irmtraud (Hgg.) (2005): Text und Sinn. Studien zur Textsyntax und Deixis im Deutschen und im Französischen. Tübingen: Stauffenburg. 291-304.

Modalfunktionen als Mittel zur Textgestaltung. In: Breindl, Eva/Gunkel, Lutz/Strecker, Bruno (Hgg.) (2005): Grammatische Untersuchungen. Analysen und Reflexionen. Gisela Zifonun zum 60. Geburtstag. Tübingen: Narr. 417-430.

Wenn die Klammer hinkt... Ein Plädoyer für das Prinzip Abgrenzung. In: Fries, Norbert/Fries, Christiane (Hgg.) (2006): Deutsche Grammatik im europäischen Dialog. Beiträge zum Kongress Krakau 2006. Onlinepublikation (www.helenevinckelroisin.fr/media/pages/files/dalmas_vinckel. pdf, Stand: 15.8.2017) (in Zusammenarbeit mit Hélène Vinckel).

Les conjonctions de subordination et l'articulation en focus et fond de la phrase complexe (en allemand et en français). In: Wlodarczyk Hélène/ Wlodarczyk, André (Hgg.) (2006): La focalisation dans les langues. Paris: L’Harmattan. 113-121 (in Zusammenarbeit mit Renate Pasch).

2008 Aspekte der Topikalisierung. In: Tarvas, Mari (Hg.) (2008): Tradition und Zukunft der Germanistik. Tallinn: Tallinna Ülikooli Kirjastus. 83-94. Konnektoren: immer noch ein verwirrendes Sammelsurium. In: Baudot, Daniel/Kauffer, Maurice (Hgg.) (2008): Wort und Text. Lexikologische und textsyntaktische Studien im Deutschen und Französischen. Festschrift für René Métrich zum 60. Geburtstag. Tübingen: Stauffenburg. 229-238.

Wie die Zeit vergeht! Welche temporalen Bezüge werden durch die sog. 'Textadverbien' markiert? In: Macris-Ehrhard, Anne-Françoise/Krumrey, Evelyn/Magnus, Gilbert (Hgg.) (2008): Temporalsemantik und Textkohärenz. Tübingen: Stauffenburg. 119-132. 
2009 Richiges Deutsch - Richtig deutsch. Normativität in französischer und deutscher Grammatik. In: Konopka, Marek/Strecker, Bruno (Hgg.) (2009): Deutsche Grammatik - Regeln, Normen, Sprachgebrauch. Berlin/New York: De Gruyter. 355-375.

Zur Bedeutungsbeschreibung von einigen Pseudokonnektoren. In: Henn-Memmesheimer, Beate/Joachim, Franz (Hgg.) (2009): Die Ordnung des Standard und die Differenzierung der Diskurse. Frankfurt a.M. u.a.: Lang. 167-178.

2010 Deutsch in Frankreich. In: Krumm, Hans-Jürgen (Hg.) (2010): Deutsch als Fremd- und Zweitsprache. Ein internationales Handbuch Band 2. Berlin: De Gruyter/Mouton. 1658-1664.

Kontrastive Analyse Französisch - Deutsch. In: Krumm, Hans-Jürgen (Hg.) (2010): Deutsch als Fremd- und Zweitsprache. Ein internationales Handbuch Band 1. Berlin: De Gruyter/Mouton. 579-585.

Topikalisierung als Mittel zur Textstrukturierung. In: Bartoszewicz, Iwona et al. (Hgg.) (2010): Germanistische Linguistik extra muros - Aufforderungen. Wrocław: Atut - Dresden: Neisse Verlag. 45-53.

Zur Bedeutungsbeschreibung von einigen Pseudo-Konnektoren. In: Henn-Memmesheimer, Beate/Franz, Joachim (Hgg.) (2010): Die Ordnung des Standards und die Differenzierung der Diskurse. Frankfurt a.M. u.a.: Lang. 167-178.

A corpus-based analysis of quasi-synonymous adjectives in German// Re-thinking synonymy: semantic sameness and similarity in languages and their description. Book of Abstracts. 28.-30.10.2010, Helsinki. 22-23 (in Zusammenarbeit mit Dmitrij Dobrovol'skij).

2011 Quasisynonymie bei Adjektiven: hervorragend und Co (eine corpusgestützte Untersuchung). In: Schmale, Günter (Hg.) (2011): Das Adjektiv. Tübingen: Stauffenburg. 173-191 (in Zusammenarbeit mit Dmitrij Dobrovol'skij).

2012 Rhetorik: eine Friedensbrücke? In: Bartoszewicz, Iwona/Dalmas, Martine/Szczek, Joanna/Tworek, Artur (Hgg.) (2012): Germanistische Linguistik im interdisziplinären Gefüge II. Wrocław: Atut - Dresden: Neisse Verlag. 13-22.

Zur Auflösung der Synkretismen bei Possessivartikeln: eine deutschfranzösische Untersuchung. In: Augustin, Hagen/Fabricius-Hansen, Cathrine (Hgg.) (2012): Flexionsmorphologie des Deutschen aus kontrastiver Sicht. Tübingen: Groos. 247-278 (in Zusammenarbeit mit Hélène Vinckel-Roisin). 
Discourse Markers in French and German: Reasons for an asymmetry. In: Taboada, Maite/Doval-Suárez, Susana/González-Álvares, Elsa (Hgg.) (2012): Functional and corpus perspectives in contrastive discourse analysis, Themenheft von: Linguistics and the Human Sciences 6/2010. 77-98. [Nachdr. in: Taboada, Maite/Doval-Suárez, Susana/GonzálezÁlvares, Elsa (Hgg.) (2013): Contrastive discourse analysis - Functional and corpus perspectives. Sheffield: Equinox Publishing. 72-93.] (in Zusammenarbeit mit Séverine Adam).

2013 Figement et pragmaticalisation. In: Yearbook of Phraseology 4. 3-21. Ihr Gesäß in guten Händen. Spielerischer Umgang mit Idiomen in 'Hohlspiegel'-Texten. In: Witze. Der Deutschunterricht 4/13. 78-81. Feste Formen, einleitend: diskursive Funktionen. In: Blachut, Edyta/ Jarosz, Jozef/Malgorzewicz, Anna/Opilowski, Roman (Hgg.) (2013): Sprachwissenschaft im Fokus germanistischer Forschung und Lehre. Wrocław: Atut - Dresden: Neisse Verlag. 55-63.

Strategien der Fokussierung: eine 'spannende' Geschichte - und eine Revidierung mancher Vorurteile. In: Adam, Séverine (Hg.) (2013): 'Informationsstrukturen' im gesteuerten Spracherwerb - Französisch-Deutsch kontrastiv. Frankfurt a.M. u.a.: Lang. 67-77.

Les constructions causatives avec mouvement en allemand: d'une saisie phraséologique à une explication constructionnelle. In: Langages, 189. 81-102 (in Zusammenarbeit mit Laurent Gautier).

Les corrélats de l'allemand. In: Inkova, Olga/Hadermann, Pascale (Hgg.) (2013): La corrélation. Aspects syntaxiques et sémantiques. Genève: Droz. 307-321.

2015 Entgleisungen und Kollisionen. In: Peschel, Corinna/Runschke, Kerstin (Hgg.) (2014): Sprachvariation und Sprachreflexion in interkulturellen Kontexten. Frankfurt a.M. u.a.: Lang. 105-116.

Bewertung durch Adjektive. Ansätze einer korpusgestützten Untersuchung zur Synonymie/Evaluation with Adjectives. Towards a Corpusbased Approach to Synonymy. In: Zeitschrift für Literaturwissenschaft und Linguistik, Volume 45, Issue 1. 12-29 (in Zusammenarbeit mit Dmitrij Dobrovol'skij, Dirk Goldhahn, Uwe Quasthoff).

2016 Germanistik - Der Blick von außen: Welche Brille darf's denn sein ? In: Szumikowski Morodo, Irene/Berit Balzer Haus (Hgg.) (2016): Die deutsche Sprache - intern und extern. Untersuchungen zu System, Vermittlung und Rezeption. Madrid: Dykinson. 15-24. 
Idiome und ihre kommunikative Leistung. Zur Theorie der informationsstrukturellen Gestaltung von Äußerungen. In: Zeitschrift für germanistische Sprachwissenschaft 2016, 44/2. 257-284 (in Zusammenarbeit mit Dmitrij Dobrovol'skij).

2017 (i.Dr.) Zur idiomatischen Komponente auffälliger kausativer Konstruktionen im Deutschen. In: Linguistik Online (erscheint Ende 2017) (in Zusammenarbeit mit Laurent Gautier).

\title{
2 Didaktisches Schaffen: Beitrag zur Konzeption und Erstellung von Lehrbüchern ${ }^{1}$
}

\author{
1996-2002 (in Zusammenarbeit mit Norbert Bisons et al.) \\ Aufwind. Classe de 6e. Paris, Didier. \\ Aufwind. Classe de 5e. Paris, Didier. \\ Aufwind. Classe de 4e. Paris, Didier. \\ Aufwind. Classe de 3e. Paris, Didier. \\ 2005-2006 (in Zusammenarbeit mit Norbert Bisons et al.) \\ Zusammen. Niveau 1. Paris, Didier. \\ Zusammen. Niveau 2. Paris, Didier. \\ 2005 (in Zusammenarbeit mit Wolf Halberstadt et al.) \\ Alternative. Classe de 1e. Paris, Didier. \\ 2006 Tout sur les verbes allemands. Paris, Larousse.
}

1 Nicht berücksichtigt sind hier die zahlreichen Beiträge über die grammatischen Fragen bei der Agrégation externe d'allemand, die in der Zeitschrift Les Nouveaux Cahiers d'allemand in den 1990er und Nuller Jahren erschienen sind. 